\title{
Understanding AGB Carbon Star Nucleosynthesis from Observations
}

\author{
C. Abia ${ }^{1}$, I. Domínguez ${ }^{1}$, R. Gallino ${ }^{2}$, M. Busso ${ }^{3}$, O. Straniero ${ }^{4}$, \\ P. de Laverny ${ }^{5}$ and G. Wallerstein 6

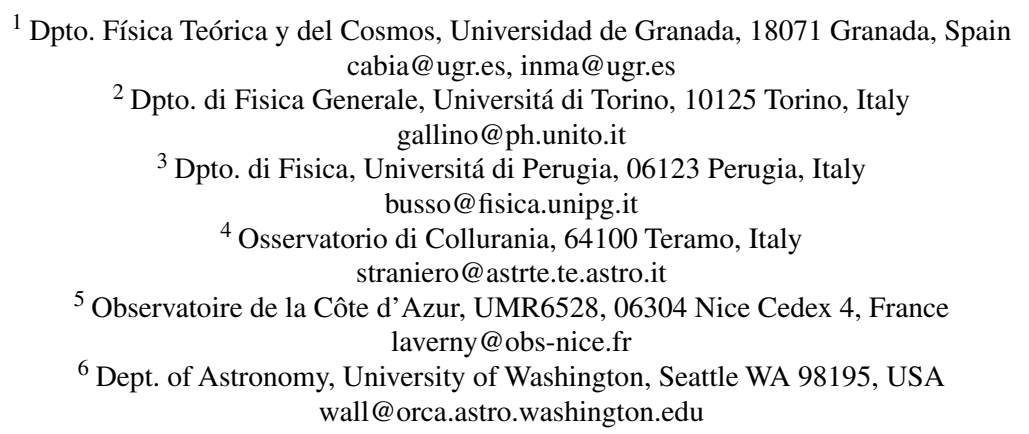 \\ Received 2003 March 20, accepted 2003 May 9
}

\begin{abstract}
Recent advances in the knowledge of the evolutionary status of asymptotic giant branch (AGB) stars and of the nucleosynthesis processes occurring in them are discussed, and used to interpret abundance determinations for s-process elements, lithium and CNO isotopes in several types of AGB stars. We focus our attention mainly on carbon-rich AGB stars. By combining these different constraints we conclude that most carbon stars in the solar neighborhood are of low mass $\left(M \leq 3 M_{\odot}\right)$, their abundances being a consequence of the operation of thermal pulses and the third dredge-up. However, the observed abundances in carbon stars of the R and J types cannot be explained by this standard scenario. These stars may not be on the AGB, but possibly in the core-He burning phases; their envelopes may have been polluted with nuclear ashes of the core-He flash, followed by $\mathrm{CNO}$ re-processing enhancing ${ }^{13} \mathrm{C}$. Observational evidence suggesting the operation of non-standard mixing mechanisms during the AGB phase is also discussed.
\end{abstract}

Keywords: nuclear reactions, nucleosynthesis, abundances — stars: abundances — stars: AGB — stars: carbon — stars: evolution

\section{Introduction}

Low and intermediate mass stars $\left(1 \leq M / M_{\odot} \leq 8\right)$ populate the giant branch in the $\mathrm{H}-\mathrm{R}$ diagram for a second time after central He exhaustion. Stars in this phase of stellar evolution are known as AGB stars. The particular internal structure of AGB stars (an inert, partially degenerate $\mathrm{CO}$ core surrounded by two burning shells, one of $\mathrm{He}$ and a second, more external, of $\mathrm{H}$, operating alternately) is the ultimate origin of the numerous chemical and spectroscopic peculiarities found among the members of this evolutionary phase. Probably the most important chemical anomaly is that many AGB stars are carbon-rich, i.e. they have a $\mathrm{C} / \mathrm{O}$ ratio (by number) $>1$ in the envelope. Since the overwhelming majority of stars are born with a $\mathrm{C} / \mathrm{O}$ ratio $<1$, this carbon enrichment must result from a deep mixing process that pollutes the envelope with carbon or, alternatively, from a transfer of carbon rich material in a binary system. In the first case the stars are usually named intrinsic carbon stars while in the second case they are named extrinsic carbon stars. Intrinsic and extrinsic O-rich AGB stars also exist, but here we will deal mainly with the zoo of carbon-rich stars.

Intrinsic carbon stars owe their carbon enrichment to the downward penetration of the convective envelope after each thermal instability, or thermal pulse (TP) (Schwarzschild \& Härm 1965) in the He-shell. This mixing phenomenon is called the third dredge-up (TDU). The simultaneous operation of TPs and TDUs along the AGB phase eventually transforms an O-rich star into a carbon star. In fact, the carbon content in the envelope is expected to increase along the spectral sequence $\mathrm{M} \rightarrow \mathrm{MS} \rightarrow \mathrm{S} \rightarrow \mathrm{SC} \rightarrow \mathrm{C}(\mathrm{N}$ type $)$, where $\mathrm{C}(\mathrm{N})$ stars show $\mathrm{C} / \mathrm{O}>1$; $\mathrm{M}$ stars have typically $\mathrm{C} / \mathrm{O} \sim 0.5$ (see e.g., Iben \& Renzini 1983; Smith \& Lambert 1990). The operation of TDU can also enrich the envelope with s-process elements, mainly produced in radiative conditions, during the interpulse period, through slow neutron capture on heavy seed nuclei. Neutrons for this process are locally released by the reaction ${ }^{13} \mathrm{C}(\alpha, n){ }^{16} \mathrm{O}$, at moderate temperatures $\left(T \leq 1.0 \times 10^{8} \mathrm{~K}\right)$. A second neutron burst is released in the TP itself, through the ${ }^{22} \mathrm{Ne}(\alpha, n)^{25} \mathrm{Mg}$ reaction, marginally activated when the temperature exceeds $3.0 \times 10^{8} \mathrm{~K}$ (see Busso, Gallino \& Wasserburg 1999, and references therein).

The importance of AGB stars and, in particular, of carbon stars is manyfold: they are excellent laboratories in which to test the theory of stellar evolution and nucleosynthesis. In fact, they are the progenitors of white dwarfs (from which, in close binary scenarios, type Ia supernovae may originate) and of planetary nebulae (PN), implying that the whole envelope containing newly synthesised metals has been previously injected into the interstellar 
medium by strong stellar winds. Carbon stars are the main producers of s-elements in the Galaxy. They are also significant contributors to ${ }^{12} \mathrm{C},{ }^{13} \mathrm{C}$ and even to rare nuclei such as ${ }^{7} \mathrm{Li}$ and ${ }^{26} \mathrm{Al}$ (see e.g., Gustafsson \& Ryde 1996; Busso et al. 2002). On the other hand, due to their large mass loss rates $\left(10^{-8}-10^{-4} M_{\odot} \mathrm{yr}^{-1}\right.$; Wallerstein \& Knapp 1998) many are surrounded by a thick circumstellar envelope. This is the place where stardust grains of amorphous carbon, $\mathrm{SiC}$ and graphite usually form. The study of these grains in the laboratory provides new insight into stellar evolution and nucleosynthesis, mixing processes in stars, the history of the Solar System, Galactic evolution and the age of the Galaxy (see e.g. Zinner 1998).

There are several types of carbon stars. They are classified spectroscopically depending mainly on the intensity of the molecular bands bearing carbon atoms $\left(\mathrm{CN}, \mathrm{C}_{2}, \mathrm{CH}\right.$, Keenan 1993) and their effective temperature (see Wallerstein \& Knapp 1998 for a detailed discussion). However, quantitative abundance and isotopic ratio determination in carbon stars of all types have allowed a clearer distinction to be made, as well as a better understanding of their nucleosynthesis histories (sometimes affected by binarity) and evolutionary status. During the last few years a number of theoretical and observational studies have added new light to this scenario. Here we discuss some of these advances, combining new theoretical and observational data. We focus our attention on giant carbon stars only. Thus we exclude from the discussion both the so called dwarf carbon stars $(\mathrm{dC}$, believed to be main sequence or subgiant stars), and the post-AGB carbon-rich stars.

\section{The Carbon Star Zoo}

\subsection{The N Stars}

The N stars, or normal carbon stars (Keenan 1993), represent a formidable challenge from the spectroscopic point of view. They show very crowded spectra due to their strong molecular band absorption and low temperatures. At optical wavelengths only a few spectral windows are suitable for abundance analysis, provided that $\mathrm{CN}$ and $\mathrm{C}_{2}$ features are included in any line list. They occupy the tip in the AGB spectral sequence $\mathrm{M} \rightarrow \mathrm{MS} \rightarrow \mathrm{S} \rightarrow \mathrm{SC} \rightarrow \mathrm{C}(\mathrm{N})$, thus they are the natural result of the continuous mixing of carbon into the envelope throughout the TDU after each TP. Despite this, the C/O ratios derived in them (see e.g. Lambert et al. 1986) do not greatly exceed unity (typically $\mathrm{C} / \mathrm{O} \leq 1.6$ ). Note that many $\mathrm{PN}$, the final outcome of the AGB phase, show $\mathrm{C} / \mathrm{O}$ ratios larger than 2 (see e.g. Parthasarathy 1999 and references therein). This apparent discrepancy may be related to different initial composition, as stellar models predict much larger $\mathrm{C} / \mathrm{O}$ ratios at lower metallicities. Moreover, this ratio is expected to increase continuously along the AGB phase until mass loss terminates the evolution; the last part of the track may, however, be occupied by infrared objects strongly enshrouded in a thick dusty envelope, so that photospheric abundances are not accessible. Hence, the sequence of $\mathrm{C} / \mathrm{O}$ values from $\mathrm{N}$ stars to $\mathrm{PN}$ may be more continuous than we can verify observationally at optical wavelengths. A critical role in this picture is played by the poorly known mass loss mechanisms at the termination of stellar evolution.

Until recently the only detailed analysis of s-elements in these stars was that by Utsumi $(1970,1985)$; however it was based on low resolution spectra. Utsumi found that $\mathrm{N}$ stars were typically of solar metallicity, presenting mean s-process element enhancements ([ls/Fe] and/or $[\mathrm{hs} / \mathrm{Fe}])^{1}$ of a factor of 10 with respect to the Sun. This figure was accepted as an extreme outcome of the s-nuclei enhancement during the AGB phase. However, more accurate studies are now available (Abia et al. 2001, 2002), based on high-resolution spectra and a more extended sample of $\mathrm{N}$ stars. This has led to strong revisions in the quantitative s-element abundances. $\mathrm{N}$ stars were confirmed to be of near solar metallicity, but they show on average $<[\mathrm{ls} / \mathrm{Fe}]>=+0.67 \pm 0.10$ and $<[\mathrm{hs} / \mathrm{Fe}]>=$ $+0.52 \pm 0.29$, which is significantly lower than that estimated by Utsumi. Present values are of the same order as those derived in the O-rich S stars (see e.g. Smith \& Lambert 1990). Contrary to superficial appearance, this is not in contradiction with the expected s-process enhancement along the AGB phase. In fact, one has to consider that the sample of $\mathrm{N}$ stars in Abia et al. (2002) shows typically $\mathrm{C} / \mathrm{O}$ ratios very slightly larger than unity. Theoretically, an $\mathrm{S}$ star $(\mathrm{C} / \mathrm{O} \sim 0.8)$ can become a $\mathrm{C}$-rich object within very few (one or two) TP and TDU episodes. This is sufficient to drastically change the appearance of the star, because above $\mathrm{C} / \mathrm{O}=1$ bands of carbon-based molecules become dominant, but the s-process element enhancement in the envelope is changed only very slightly, in agreement with observations. We might therefore speculate that the $\mathrm{N}$ stars analysed by Abia et al. have just recently become C-rich! Later, as the star evolves in the AGB phase and TPs and TDUs continue operating, more carbon and s-elements are dredged-up into the envelope. Eventually, the $\mathrm{C} / \mathrm{O}$ ratio may exceed unity by a consistent amount, before stellar winds terminate the evolution; but, as said, the simultaneous increase of mass loss rates forms a thick and dusty circumstellar envelope obscuring the star at optical wavelengths. This scenario might explain not only the already mentioned large $\mathrm{C}$ abundances observed in some $\mathrm{PN}$, but also the apparent gap in the level of s-element and carbon enhancement between $\mathrm{N}$ stars and post-AGB stars (Van Winckel \& Reyniers 2000). We cannot 'see' carbon stars with a $\mathrm{C} / \mathrm{O}$ ratio largely exceeding unity.

Figure 1 shows a comparison of the [hs/ls] ratio (a parameter sensitive to the neutron exposure) versus the metallicity in the sample studied in Abia et al. (2002) with theoretical predictions of s-process nucleosynthesis in AGB stars (Gallino et al. 1998; Busso et al. 2001). The continuous line represents the prediction for a $1.5 M_{\odot}$ stellar model at $\mathrm{C} / \mathrm{O} \sim 1$ assuming that the mass of ${ }^{13} \mathrm{C}$ burned

\footnotetext{
${ }^{1}$ We adopt the usual notation $[\mathrm{X} / \mathrm{Y}] \equiv \log (\mathrm{X} / \mathrm{Y})$ programstar $-\log$ $(\mathrm{X} / \mathrm{Y})_{\text {comparisonstar }}$ for the stellar value of any element ratio $\mathrm{X} / \mathrm{Y}$. In the following we use 'ls' to refer to the light mass s-elements $\mathrm{Y}$ and $\mathrm{Zr}$, and 'hs' to denote the high mass s-elements $\mathrm{Ba}, \mathrm{Nd}, \mathrm{La}$ and $\mathrm{Sm}$.
} 


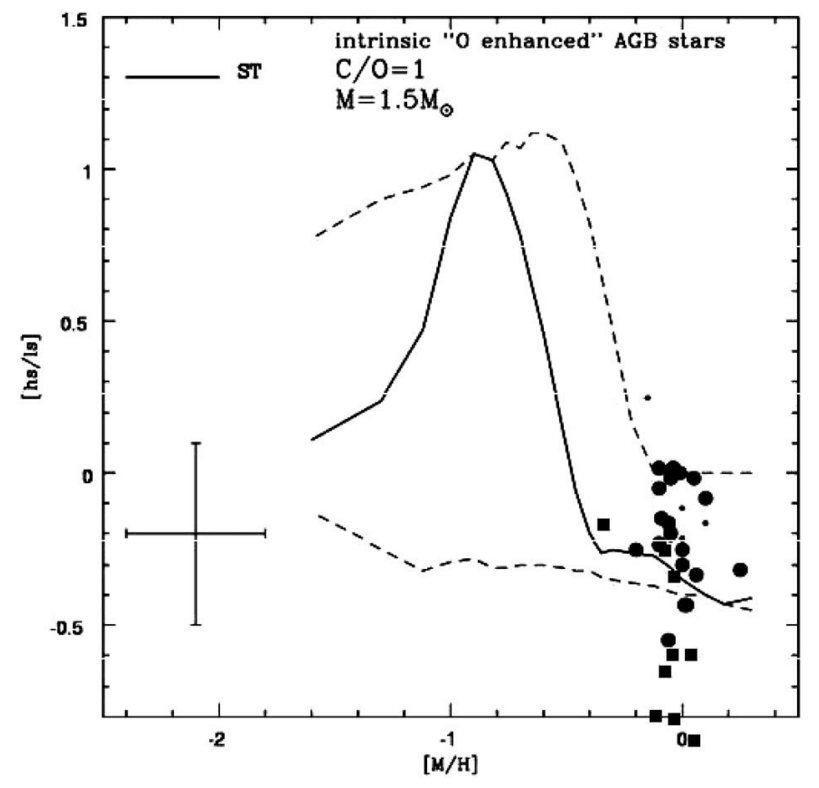

Figure 1 Comparison of the observed mean high-mass s-element (Ba, La, Nd, Sm) (large circles; Abia et al. 2002) to the low-mass s-element $(\mathrm{Y}, \mathrm{Zr})$ ratio $[\mathrm{hs} / \mathrm{ls}]$ in $\mathrm{N}$ stars against metallicity with theoretical predictions for a $1.5 M_{\odot}$ TP-AGB star. The theoretical predictions shown are for $\mathrm{O}$-enhanced stellar models at $\mathrm{C} / \mathrm{O}=1$ (see text). The upper and lower curves (dashed lines) limit the region allowed by the models according to the different ${ }^{13} \mathrm{C}$-pocket choices (see Busso et al. 2001). Note that several stars coincide at the same data point. Small circles are stars with very uncertain abundances. Black squares are the old data by Utsumi (1985).

per cycle is $4 \times 10^{-6} M_{\odot}$ (the so-called standard, or ST case, in the quoted models). Dashed lines limit the area allowed by theoretical models according to the different choices for the ${ }^{13} \mathrm{C}$-pocket (Straniero et al. 1995). Theoretical models are computed assuming an initial oxygen content derived from the $[\mathrm{O} / \mathrm{Fe}]$ trend observed in field stars at different metallicities. As a comparison, the [hs/ls] ratios obtained by Utsumi (1985) are also shown. Theory and observations are now in better agreement. A similar comparison with theoretical predictions for a $3 M_{\odot}$ or $5 M_{\odot}$ AGB model (see Figure 2) does not allow us to draw conclusions about the typical mass of $\mathrm{N}$ stars. This can be done, however, using the properties of the s-process path at reaction branchings. In low mass stars (LMS) the major neutron source is ${ }^{13} \mathrm{C}$-burning at low temperatures $(\approx 8 \mathrm{keV})$, as a consequence of the formation of a ${ }^{13} \mathrm{C}$-rich pocket in the radiative intershell region. In the rarer AGB stars of intermediate mass (IMS) $\left(M \geq 4 M_{\odot}\right)$, ${ }^{22} \mathrm{Ne}$ would instead be favoured as a neutron donor, by the higher temperature in thermal pulses. Moreover, the mass of the $\mathrm{He}$ intershell, that is the region between the $\mathrm{H}$ shell and the He shell, is one order of magnitude less in intermediate mass stars than in lower mass AGBs. This makes the s-process elements built in the TP to be less diluted by convective motions. Furthermore, the ${ }^{13} \mathrm{C}$ pocket and the hydrodynamic penetration of protons in the top layers of the He intershell is less favoured. Our standard case for the choice of the ${ }^{13} \mathrm{C}$-pocket in the intermediate mass case is consequently less efficient by an order of magnitude than

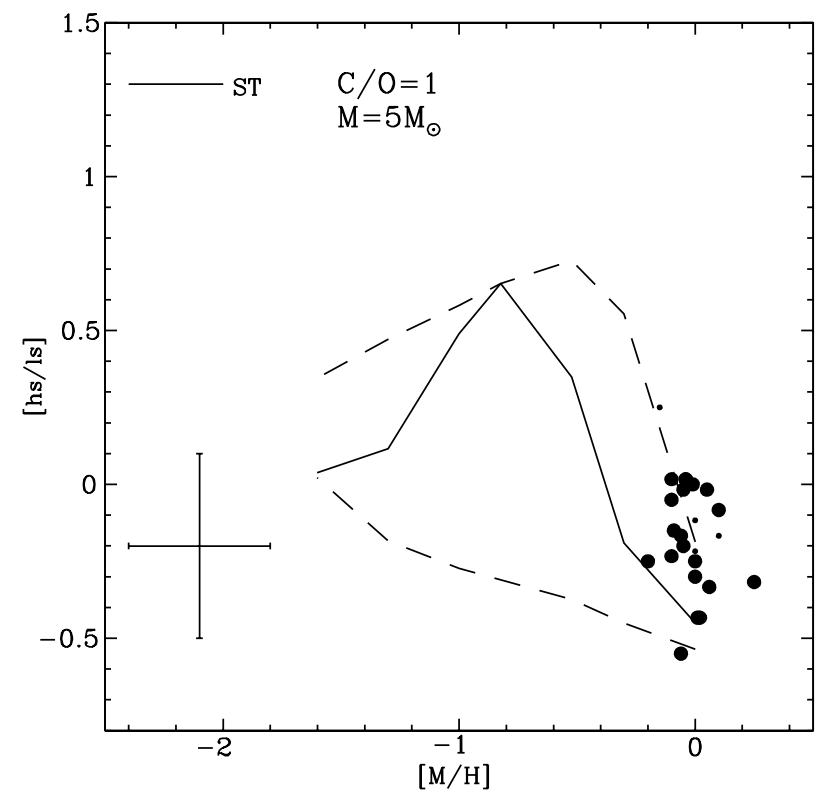

Figure 2 Same as Fig. 1 compared with theoretical predictions for a $5 M_{\odot}$ TP-AGB star. Note that in this case theoretical models cannot form carbon stars for metallicity larger than solar.

the ST choice in low mass AGBs (see Busso et al. 2001 for a detailed description of the choices of the ${ }^{13} \mathrm{C}$ pocket mass in each case). Because of the very different neutron density provided by the two neutron-producing reactions, different compositions are expected from them, especially for the ratio between $\mathrm{Rb}$ (whose isotope ${ }^{87} \mathrm{Rb}$ is strongly fed only at high neutron densities) and its neighbors Sr, Y and $\mathrm{Zr}$. It is on this basis that Abia et al. (2001) showed that $\mathrm{N}$ stars typically have a low mass $\left(M \leq 3 M_{\odot}\right)$.

Another interesting result, connected also with the actual mass of $\mathrm{N}$ stars, is the observed ${ }^{12} \mathrm{C} /{ }^{13} \mathrm{C}$ ratio. Currently there is still some discussion concerning the typical value of this ratio in $\mathrm{N}$ stars. Some authors claim that the mean value is around 60 (Lambert et al. 1986; de Laverny \& Gustafsson 1998, 1999; Schöier \& Olofsson 2000), some that it is significantly lower, $\sim 30$ (Ohnaka \& Tsuji 1996, 1998). Although most of this discrepancy may have a spectroscopic origin (different choices of the ${ }^{12} \mathrm{C} /{ }^{13} \mathrm{C}$ feature indicator, model atmospheres, interpretation of the differences between circumstellar and photospheric ratios etc.), even considering the observational uncertainties a significant number of $\mathrm{N}$ stars have ${ }^{12} \mathrm{C} /{ }^{13} \mathrm{C}<25$ (Abia et al. 2002). Standard AGB models for LMS and IMS cannot account for these low isotopic ratios at $\mathrm{C} / \mathrm{O} \sim 1$ (see Figure 3). This is so even when the effects of an extra-mixing mechanism along the RGB (sometimes named the cool bottom process) are considered, which reduce this ratio (see e.g. Wasserburg, Boothroyd, \& Sackmann 1995; Weiss, Denissenkov, \& Charbonnel 2000). Actually, even accepting initial ${ }^{12} \mathrm{C} /{ }^{13} \mathrm{C}$ values for the AGB phase as low as those observed in many low mass RGB stars (in the range 5-10, see e.g. Gilroy \& Brown 1991; Gratton et al. 2000), this ratio is then increased strongly by TDU. The low ${ }^{12} \mathrm{C} /{ }^{13} \mathrm{C}$ ratios in $\mathrm{N}$ stars require that a non-standard deep mixing also takes 


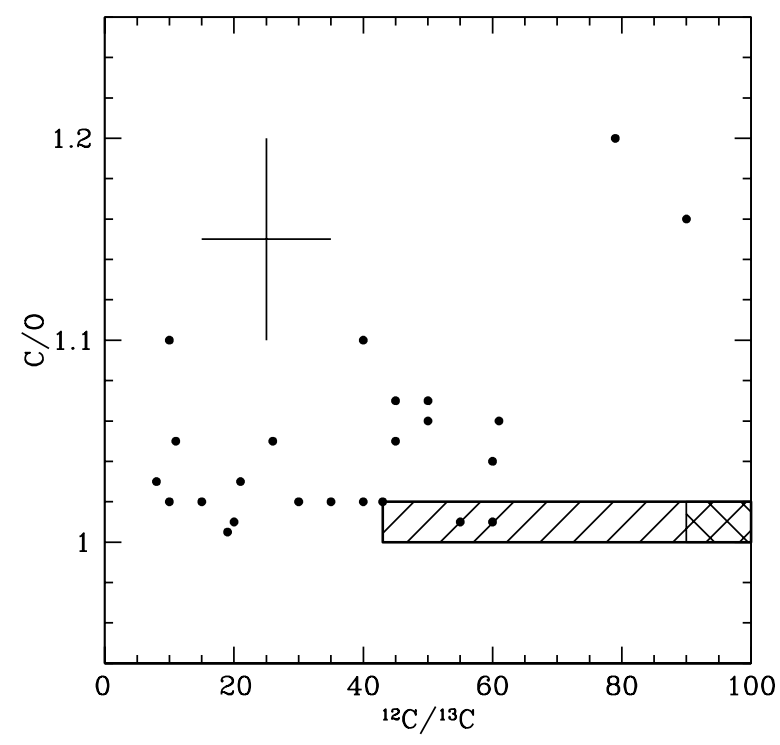

Figure 3 The $\mathrm{C} / \mathrm{O}$ vs. ${ }^{12} \mathrm{C} /{ }^{13} \mathrm{C}$ ratio in $\mathrm{N}$ stars. Single dashed box is the range of carbon isotopic ratios predicted in low mass AGB stars at $\mathrm{C} / \mathrm{O} \sim 1$. Double dashed box is the same for intermediate mass AGB stars. These computations assume a ${ }^{12} \mathrm{C} /{ }^{13} \mathrm{C} \sim 12$ after the first dredge-up as observed in many low mass RGB stars (for details on these computations see Abia et al. 2001). A significant number of $\mathrm{N}$ stars show ${ }^{12} \mathrm{C} /{ }^{13} \mathrm{C}$ lower than theoretical predictions in the AGB phase. An additional episode of non-standard mixing seems also to be required in $\mathrm{N}$ stars. The error bar in the ${ }^{12} \mathrm{C} /{ }^{13} \mathrm{C}$ ratio is an average value; the lower this ratio, the lower the actual error.

place on the AGB itself, preferably of low mass, as shown by Boothroyd \& Sackmann (1999). This mechanism could also account for the oxygen isotopic anomalies observed in some $\mathrm{N}$ stars with $\mathrm{C} / \mathrm{O} \sim 1$ (Harris et al. 1987) and for the carbon and nitrogen isotopic ratios measured in mainstream SiC grains (see e.g Nittler et al. 1995; Amari et al. 1997). The nature of this extra-mixing mechanism is still unknown, though diffusion and/or rotational shear could play a role. Parametric models of its occurrence (Nollett, Busso, \& Wasserburg 2003) show however that the regimes needed to fit observations in $\mathrm{N}$ stars are very easily reached by limited mass circulations, whatever the physical origin may be.

More evidence of the existence of non-standard mixing mechanism(s) in $\mathrm{N}$ stars is provided by the lithium (Li) observations. It is well known that $2-3 \%$ of $\mathrm{C}$ stars show Li enhancements; a few are super Li-rich (Abia et al. 1993). Li can be produced in AGB stars by the operation of the Cameron \& Fowler (1971) mechanism at the bottom of a moderately hot $\left(T>30 \times 10^{6} \mathrm{~K}\right)$ convective envelope (Sackmann \& Boothroyd 1992). These temperatures are reached in IMS AGB stars (the exact limit depending on the metallicity) where, in addition, the proton captures on carbon at the bottom of the convective envelope may prevent the formation of a carbon star. However, the evidence that $\mathrm{N}$ stars are mostly of low mass $\left(M \leq 3 M_{\odot}\right)$ does not make these hot bottom burning (HBB) models viable. How can Li be produced in low mass objects? Some of the Li-rich N stars are also ${ }^{13} \mathrm{C}$-rich. Charbonnel (1995) and
Charbonnel \& Balachandran (2000) suggest also in this case an explanation in terms of deep mixing for the high $\mathrm{Li}$ abundances found in low mass RGB stars. A similar mechanism might work in the AGB phase.

To conclude this section, let us address the problem of the intrinsic or extrinsic nature of $\mathrm{N}$ stars. Binarity can be tested by searching for radial velocity variations, though one can never exclude the possibility of very long orbital periods; in any case, no such variations have been clearly detected to date (see e.g. Barnbaum et al. 1995). On the other hand, note that it is very difficult to differentiate between such velocity variations and those induced by atmospheric motions due to the stellar pulsations (these stars are long period variables). A more direct answer can be obtained from the detection of the radioactive s-nuclide ${ }^{99} \mathrm{Tc}\left(\tau_{1 / 2}=2.1 \times 10^{5} \mathrm{yr}\right)$, an unambiguous signature of the operation of an internal nucleosynthesis and mixing process in the star. This is not an easy task in AGB stars as the strongest Tc lines are placed in a very crowded spectral region $(\lambda \sim 4260 \AA)$ where, in addition, $N$ stars are quite faint (Little et al. 1987). Using the $\lambda 5924 \AA$ Tc I line, Abia et al. $(2001,2002)$ detected Tc in $\sim 60 \%$ of the members in their sample of $30 \mathrm{~N}$ stars. Due to the difficult analysis of this Tc line, the presence of Tc in the rest of the sample cannot be excluded. The existence of an infrared excess related to dust can be used as an additional test. Thermally pulsing AGB stars (i.e. intrinsic AGB stars) are usually high mass-losing objects showing mass loss rates from $10^{-7}$ up to $10^{-4} \mathrm{M}_{\odot} \mathrm{yr}^{-1}$ (Wallerstein \& Knapp 1998). These huge mass-loss rates favour the formation of a cool circumstellar envelope where dust can easily form. As a consequence of the dust formation, intrinsic AGB stars are expected to have an infrared colour excess that can be measured, e.g. by the flux ratio $F(12 \mu \mathrm{m}) / F(2.2 \mu \mathrm{m})$. Thus, the detection of this colour excess would be an indication of the presence of a high mass loss rate and, therefore, that the star is very probably suffering from thermal pulses which is evidence that the star has an intrinsic nature. Jorissen et al. (1993) showed that S-stars with Tc (intrinsic AGBs) generally have this ratio $>0.1$. In fact, $\sim 95 \%$ of the $\mathrm{N}$ stars in the Abia et al. (2002) sample exceed this limit.

A final test can be performed by modelling the mass transfer of carbon rich material in a binary system to try to form an extrinsic carbon star. This can be expressed in terms of a dilution factor $f$, which is the ratio of the transferred mass $\left(M_{\mathrm{AGB}}^{\mathrm{tr}}\right)$ to the secondary star envelope mass, with the original composition $\left(M_{\text {comp }}^{\text {envini }}\right)$ preserved. We must impose the condition that the envelope of the secondary star (the observed carbon star in extrinsic objects) reaches a C/O ratio of 1 (as observed, see Figure 3). Even assuming that mass transfer occurs at the last TP of the primary star (in order to maximise the $\mathrm{C} / \mathrm{O}$ ratio in the transferred mass), and that the secondary is on the main sequence (for which the absence of a convective envelope requires contamination of only a small layer), Abia et al. (2002) found that, for metallicities not far from solar, it is impossible to produce an extrinsic carbon star. 
Table 1. Predicted C/O Ratio and $f$ Value in Extrinsic AGB Stars

\begin{tabular}{|c|c|c|c|c|c|c|}
\hline \multirow[t]{2}{*}[\mathrm{Fe}/\mathrm{H}]{} & \multicolumn{2}{|l|}{$1.5 M_{\odot}$} & \multicolumn{2}{|c|}{$3.0 M_{\odot}$} & \multicolumn{2}{|l|}{$5.0 M_{\odot}$} \\
\hline & $(\mathrm{C} / \mathrm{O})_{\mathrm{AGB}}^{\text {lastTDU }}$ & $f$ & $(\mathrm{C} / \mathrm{O})_{\mathrm{AGB}}^{\mathrm{lastTDU}}$ & $f$ & $(\mathrm{C} / \mathrm{O})_{\mathrm{AGB}}^{\mathrm{lastTDU}}$ & $f$ \\
\hline+0.10 & 1.32 & 2.17 & 1.05 & 13.88 & 1.21 & 3.30 \\
\hline+0.00 & 1.45 & 1.60 & 1.14 & 5.14 & 1.32 & 2.25 \\
\hline-0.12 & 1.65 & 1.15 & 1.28 & 2.68 & 1.49 & 1.53 \\
\hline-0.30 & 2.00 & 0.79 & 1.54 & 1.46 & 1.80 & 0.98 \\
\hline-0.40 & 4.28 & 0.25 & 2.97 & 0.41 & 2.01 & 0.80 \\
\hline-0.52 & 5.02 & 0.21 & 3.78 & 0.30 & 2.32 & 0.63 \\
\hline-0.60 & 5.55 & 0.18 & 3.84 & 0.29 & 2.55 & 0.54 \\
\hline-0.70 & 6.28 & 0.16 & 4.34 & 0.26 & 2.87 & 0.46 \\
\hline-0.82 & 7.36 & 0.14 & 5.54 & 0.19 & 3.34 & 0.37 \\
\hline-1.00 & 9.18 & 0.11 & 6.90 & 0.15 & 4.13 & 0.28 \\
\hline-1.30 & 13.29 & 0.07 & 9.98 & 0.10 & 5.93 & 0.19 \\
\hline-1.60 & 33.08 & 0.03 & 24.54 & 0.04 & 8.34 & 0.13 \\
\hline
\end{tabular}

This is shown in Table 1 where we report, for the 1.5, 3 and $5 M_{\odot}$ models at different metallicities (column 1), the final $\mathrm{C} / \mathrm{O}$ ratio of the intrinsic AGB star (primary component) at the last thermal pulse that experienced TDU $\left((\mathrm{C} / \mathrm{O})_{\mathrm{AGB}}^{\text {lastTDU }}\right)$ and the corresponding dilution ratio $f$. It is important to note that the $f$ values in Table 1 correspond to very extreme cases; substantially lower values of the $f$ parameter would be more realistic (see Abia et al. 2002 for details $)^{2}$. In any case, from a comparison with the calculated $f$ values reported in Table 1, it is clear that it would be practically impossible to form extrinsic carbon stars for solar metallicities. We suggest that the maximum metallicity of an extrinsic carbon star should be of the order of $[\mathrm{Fe} / \mathrm{H}] \sim-0.4$ to -0.3 .

From Table 1 it is also obvious that at lower metallicities, an extrinsic carbon star can be easily formed for all the stellar mass range. Indeed, observations show that many of the metal-poor carbon stars belong to binary systems (see below). Thus, even considering the uncertainty in the metallicity of $\mathrm{N}$ stars $( \pm 0.3 \mathrm{dex})$, this simple result prevents the vast majority of them from being considered as extrinsic carbon stars. This gives strong support to the indications already obtained from the observational criteria discussed above.

\subsection{The SC Stars}

Carbon stars of type SC are AGB stars with a C/O ratio very close to unity, within $1 \%$ or less. They have spectral characteristics that are used to distinguish them from the normal N stars, for example the huge intensity of the $\mathrm{NaD}$ lines (see Keenan \& Boeshaar 1980 for a complete description of their spectral properties). Because the $\mathrm{C} / \mathrm{O}$ ratio is very close to one, the near-balance of oxygen and carbon in the atmosphere makes oxide bands and carbon molecules very weak, thus revealing a plethora of atomic

\footnotetext{
${ }^{2}$ Note that according to the FRANEC code $M_{\mathrm{AGB}}^{\mathrm{tr}}$ is limited to a maximun value of $\sim 0.5 M_{\odot}$. Obviously, for a given initial mass of the secondary, $M_{\text {comp }}^{\text {envini is also limited. }}$
}

lines (Wallerstein 1989) which makes the analysis of these stars easier (in principle).

In the standard scenario of spectral evolution on the AGB phase, SC stars are placed just before $\mathrm{N}$ stars (see above). The operation of the TDU is accepted to be responsible for their carbon enhancement. The $\mathrm{C} / \mathrm{O}$ $\approx 1$ might be the reason why there are very few AGB stars classified as SC type. Accepting this scenario, one would expect very similar chemical characteristics to those of $\mathrm{N}$ stars, however this is not always the case. SC stars have on average lower ${ }^{12} \mathrm{C} /{ }^{13} \mathrm{C}$ ratios (Ohnaka \& Tsuji 1996) than the $\mathrm{N}$ stars (some SC stars have near equilibrium ratios). Concerning s-elements Abia \& Wallerstein (1998) found a mean s-element enhancement $<[\mathrm{h} / \mathrm{Fe}]>=+1.06 \pm 0.27$, which is a factor 2-3 higher than that found in $\mathrm{N}$ stars, and placed upper limits on the Tc content in their sample. This indicates that they are probably intrinsic stars. However, this result might be in contradiction with the idea that SC stars are in a less advanced stage on the AGB than N stars; one would expect similar or slightly smaller s-element enhancements in SC stars. Subsequently, Abia et al. (2001) showed that the s-element content in SC and N stars can be put into agreement (between error bars) when the same grid of model atmospheres is used in the analysis. In fact, when $\mathrm{C} / \mathrm{O}$ approaches unity, the computation of molecular continuum opacity in model atmospheres is not trivial and different assumptions can lead to very different model structure, particularly in the outer layers of the atmosphere where most of the available atomic lines form. As an example, we checked that molecular equilibrium calculations with different model atmospheres for SC stars (Johnson 1982; Hauschildt et al. 1999; Pavlenko 2003; Plez, private communication) show that, for a given line, the ${ }^{12} \mathrm{C} /{ }^{13} \mathrm{C}$ ratio can vary from 25 to 150 as $\mathrm{C} / \mathrm{O}$ varies from 0.98 to 1.02 depending on the model atmosphere used. This fact is a clear indication of the large uncertainties involved in the chemical analysis of AGB stars, the use of realistic model atmospheres being a critical point (Plez 1999).

The situation is nevertheless far from clear. Recently Bergeat, Knapik, \& Rutily (2002) studied the luminosity function of Galactic carbon stars. These authors find that all the SC stars in their sample (five stars) are among the brighter carbon stars, $\left(-7.0 \leq M_{\text {bol }} \leq-5.5\right)$. When comparing their position in the $\mathrm{H}-\mathrm{R}$ diagram with standard evolutionary tracks with $Z=0.02$, they correspond to IMS. These stars experience $\mathrm{HBB}$, the main consequence of which is the decrease of the $\mathrm{C} / \mathrm{O}$ ratio and the increase of the ${ }^{12} \mathrm{C} /{ }^{13} \mathrm{C}$ ratio in the envelope. Typically stars suffering $\mathrm{HBB}$ are O-rich stars, not carbon stars. However such stars can become C-rich for a short period of time (Frost et al. 1998) at the very end of their evolution on the AGB. Due to the high mass-loss rate at this stage, HBB is inhibited while TDU is still going on. Eventually, the star becomes a very bright carbon star surrounded by a thick circumstellar envelope. Such obscured carbon stars have been observed in the LMC (van Loon et al. 1998). Then, if we identify SC stars with these objects, this might 
explain why they show larger s-element enhancements than $\mathrm{N}$ stars: they are in a more advanced stage on the $\mathrm{AGB}$, thus they have experienced more TDU episodes. The relatively low ${ }^{12} \mathrm{C} /{ }^{13} \mathrm{C}$ ratios observed, as well as the $\mathrm{C} / \mathrm{O}$ ratio very close to one, may also be explained by the operation of HBB. This scenario has at least one consequence: SC stars should present very high mass-loss rates. To test this idea we have derived the mass loss rates from the $\mathrm{K}-[12]$ index and the calibration by Le Bertre (1997) in a sample of 20 Galactic SC stars (including those studied by Bergeat, Knapik, \& Rutily 2002). We find mass loss rates very similar to those of $\mathrm{N}$ stars. Also, using the parallaxes reprocessed by Pourbaix et al. (2003), we find similar luminosities as the N-type giants. Therefore, we advance some doubts on the evolutionary status of SC suggested by Bergeat et al. (2002). We note, however, that our statement is based on very low statistics and on an uncertain calibration of mass-loss (see above). A chemical reanalysis of SC stars in a more extended sample is urgently needed.

\subsection{The R Stars}

From the spectroscopic point of view many $\mathrm{R}$ stars are indistinguishable from $\mathrm{N}$ stars. A more detailed analysis allows us to differentiate between the early (hot) R stars (spectral types R0-R4), which look similar to the normal K giants, and the late (cool) R stars (R5-R8), more similar to M stars. Early studies by Vandervort (1958) concerning their kinematics and luminosity properties have been confirmed recently by Knapp, Pourbaix, \& Jorissen (2001) and Pourbaix et al. (2003). By reprocessing Hipparcos parallaxes of Galactic carbon stars these authors have shown that hot $\mathrm{R}$ stars are concentrated in the $\mathrm{H}-\mathrm{R}$ diagram in a location similar to that occupied by redclump stars in globular clusters: $-2.5 \leq M_{\mathrm{K}} \leq-0.5,2 \leq$ $(V-K)_{\mathrm{o}} \leq 3$, i.e. they are core-He burning stars and not AGB stars. Their mean $\mathrm{M}_{K}$ magnitude is five magnitudes fainter than the typical $\mathrm{N}$ star value. This is in agreement with the observational fact that the overwhelming majority of hot R stars do not show any variability, nor are they significant mass-loss stars as judged from the absence of $12 \mu \mathrm{m}$ excess (Le Bertre 1987), two characteristics that are typical of AGB stars. On the other hand, their kinematics are typical of thick-disk stars, indicating also that they probably belong to a different (less massive) population than the $\mathrm{N}$ stars showing thin-disk kinematics (see e.g. Claussen et al. 1987). How then can R stars become carbon rich? Mass transfer in a binary system is the first possibility, but a systematic study in a sample of 22 hot R stars by McClure (1997) showed no sign of binarity (although he concluded just the opposite: the fact that no one $\mathrm{R}$ star is binary most likely demostrated that all $\mathrm{R}$ stars were binaries in the past and they just merged). On the contrary, their location in the red clump strongly supports the suggestion by Cole \& Deupree (1980) that these stars have enriched the envelope with carbon during and/or after the He-core flash. In this case, they would be intrinsic carbon stars. The sole detailed chemical analysis by Dominy (1984) supports this idea: hot R stars do not show s-process element enhancements as would be expected in normal AGB carbon stars. Their additional chemical properties are low ${ }^{12} \mathrm{C} /{ }^{13} \mathrm{C}$ ratios $(<10)$, normal oxygen isotopic ratios, near solar metallicity and the absence of $\mathrm{Li}$; these put strong constraints on any nonstandard mixing mechanism suitable to transport carbon into the envelope during the He-flash.

With regard to late or cool R stars, Knapp, Pourbaix, $\&$ Jorissen (2001) claim that these stars are on average brighter than their R-hot counterparts and occupy a location on the $\mathrm{H}-\mathrm{R}$ diagram similar to $\mathrm{N}$ stars, $(V-K)_{\mathrm{o}}>4$. The fact that R-cool stars usually show some type of variability and suffer from substantial mass loss (as indicated by the $12 \mu \mathrm{m}$ excess), is used by these authors to conclude that these stars instead mark the bottom of the AGB and are thus related, or even identical, to the $N$ stars. However, we note that the statistics used are rather low $(\sim 20$ R-cool stars studied against $\sim 60 \mathrm{R}$ hot), and that some $\mathrm{R}$-cool stars in the sample have a luminosity intermediate between R-hot and $\mathrm{N}$ stars. This might indicate instead that R-cool stars are just the more evolved (cooler) descendants of the R-hot stars. We believe that a full chemical analysis of R-cool stars is needed before a final answer to their evolutionary status might be advanced. This should include in particular the search for Tc and s-process elements, signatures of TP-AGB stars. Note that a $\sim 1 M_{\odot}$ star with solar metallicity (which suffers the He-flash) does not have TDU, and therefore does not dredge-up either carbon or s-process elements into the envelope, and is indistinguishable in luminosity on the AGB from a typical (more massive) $\mathrm{N}$ star. We note also that the $\mathrm{K}-[12]$ criterion to estimate the mass-loss rate must be taken with some care since it is valid only for groups of stars, on average. This is so due to the large error bars of IRAS, and also because the $\mathrm{K}$ band is affected by the photosphere, the actual colour depending on the variability phase.

\subsection{The J Stars}

Carbon stars of type $\mathrm{J}$ probably represent the greatest challenge. These stars show very strong $\mathrm{CN}$ and $\mathrm{C}_{2}$ bands and crowded spectra. They are easily recognised from the intensity of these bands formed with ${ }^{13} \mathrm{C}$ atoms, since their main chemical property is the very low ${ }^{12} \mathrm{C} /{ }^{13} \mathrm{C}$ ratio, close to the $\mathrm{CNO}$ cycle equilibrium value $(\sim 3)$. Spectroscopically, they are very similar to the late (cool) R-type stars. Chemically they present very similar abundance patterns as the R-hot stars (Abia \& Isern 2000; Dominy 1984), differing only in Li. It is very frequent to find Li enhancements among the J-type stars. In a sample of 15 Galactic J stars, Abia \& Isern (1997) found Li enhancements in $85 \%$ of them. However, very few are super Li-rich stars $(12+\log \mathrm{N}(\mathrm{Li}) / \mathrm{N}(\mathrm{H}) \geq 4)$. This high frequency of $\mathrm{Li}$ enhancement is also observed in $\mathrm{J}$ stars of the LMC, but none of them has been classified as super Li-rich (Hatzidimitriou et al. 2003). 
Abia \& Isern (2000) derived s-element abundances in a sample of Galactic J stars. They have near solar metallicity; the mean heavy element enhancement in the sample being $<[\mathrm{h} / \mathrm{Fe}]>=+0.13 \pm 0.12$, which is compatible with no s-enrichment. Tc is also absent in these stars; generous upper limits were placed on only two stars in the sample. The absence of s-process element overabundances and Tc indicates that these stars are not suffering from TDU. However, since they have similar luminosities (Pourbaix et al. 2003) to the normal N stars and also show some kind of variability, this would indicate that they are in the AGB phase ${ }^{3}$.

This is also observed in $\mathbf{J}$ stars of the LMC (Hatzidimitriou et al. 2003) although, in this case, the lower metallicity may play a role. The location of $\mathrm{J}$ stars in the AGB spectral sequence is far from clear. In fact, other authors have located them in a different evolutionary sequence from that of the ordinary carbon stars (see e.g. LorenzMartins 1996). Again, one might suggest the mass transfer scenario as the origin of the carbon enhancement. Leaving apart the fact that no radial velocity variations have been detected in these stars and the difficulty in creating a carbon star by mass transfer at solar metallicity (see Table 1), it is not clear how Li can survive in the mass transfer episode. Taking all this information together, an alternative scenario might emerge: $\mathrm{R}$ (hot and cool) and $\mathrm{J}$ stars represent about $\sim 15 \%$ of Galactic carbon stars of all types. This is a significant number, which can be understood if these stars are preferably of low mass. J stars might be the descendants of $\mathrm{R}$ stars. They are low mass objects $\left(M \sim 1-1.2 M_{\odot}\right)$ in such a way that they enter into the AGB but do not suffer from TDU because of their very low mass. Similar to R stars, the carbon enhancement of $\mathbf{J}$ stars is due to mixing during the He-flash, possibly triggered by a rapidly rotating core (Mengel \& Gross 1976). During the mixing episode, the nature of which is not known, the temperature is high enough to burn ${ }^{12} \mathrm{C}$ into ${ }^{13} \mathrm{C}$ but not to produce s-elements. Subsequently, when the star evolves and ascends the AGB, this mixing mechanism (or any other) is also able to produce $\mathrm{Li}$ and the star becomes a $\mathbf{J}$ star. On the AGB, J stars are hard to distinguish from the normal $\mathrm{N}$ stars in terms of luminosity as a $\sim 1 M_{\odot}$ star has similar luminosity on the AGB to a $\sim 2-3 M_{\odot}$ star that has not evolved very much. Obviously, theoretical and observational work is needed to test this scenario.

\subsection{The CH Stars}

Of the various types of carbon stars, the (subgiant and giant) $\mathrm{CH}$ stars are probably the easiest to analyse because they are metal-poor (Luck \& Bond 1991; Vanture 1992a,b) with $[\mathrm{Fe} / \mathrm{H}]$ values ranging from -0.5 to -2.0 . They usually show high velocities indicating an old stellar population. The reprocessing of the Hipparcos parallaxes by Pourbaix et al. (2003) definitely placed the giant $\mathrm{CH}$

${ }^{3}$ Note however, that there are some hints indicating that Galactic J stars are in the mean less luminous than N stars (Abia \& Isern 2000). stars in the horizontal-branch phase (nevertheless, some exceptions exist such as the $\mathrm{CH}$ stars in Omega Cen). As they are not TP-AGB stars, the mass transfer scenario has been assumed to explain their carbon enhancement and chemical peculiarities. Indeed, radial velocities by McClure \& Woodsworth (1990) showed that most of CH stars are binaries with companions that are now presumably white dwarfs. As we showed in the previous sections (see Table 1), the formation of carbon stars by mass transfer is favoured at low metallicities. According to Vanture (1992c), Kipper \& Jørgensen (1994) and Kipper et al. (1996), the s-process elements in giant $\mathrm{CH}$ stars are enhanced by factors of 10-100, with the hs (Ba through $\mathrm{Sm}$ ) species more enhanced than the ls species. This is in perfect agreement with the current theoretical modelling of the s-process in stars and its dependence on the metallicity (Busso, Gallino, \& Wasserburg 1999; Busso et al. 2001). Figures 4 and 5 show theoretical reproductions of the observed abundances in the $\mathrm{CH}$ stars HD 189711 and HD187216 (Abia et al. 2003). In both figures two possible fits are shown according to whether the star is an intrinsic or extrinsic (binary) $\mathrm{CH}$ star. In the second case, the abundances in the envelope have been computed by simply assuming a dilution factor due to the transfer of mass from the primary. The instrinsic solution is obtained when the AGB star has reached a quite limited number of third dredge-up episodes. The extrinsic solution is adopted instead under the hypothesis that the primary AGB suffered envelope mass transfer on the companion (the presently observed star) while at a more advanced phase. There, the s-process abundances at the moment of mass transfer were more enriched due to further TDU phenomena, with a relatively higher production in the heavy $(A>130)$ s-elements (in particular the s-elements at the Ba-peak at neutron magic $N=50$ ) compared to the lighter elements, in particular the s-elements at the Zr-peak at neutron magic $N=82$. After dilution with the envelope of the unperturbed companion, the net effect on all s-elements with $A>130$ is virtually zero with respect to the intrinsic solution, while the lighther s-elements appear to be somewhat less produced as compared with the intrinsic solution. The aim of these plots is to show that extremely similar results can be obtained in the two cases, so that we cannot, on nuclear grounds, decide whether the star is intrinsic or not.

The ${ }^{12} \mathrm{C} /{ }^{13} \mathrm{C}$ ratios derived in giant $\mathrm{CH}$ stars (Vanture 1992a,b; Aoki \& Tsuji 1997) might confirm that different evolutionary and mixing histories exist among these stars. Many $\mathrm{CH}$ stars show carbon ratios from 3 to 10 , i.e. near the equilibrium value. Most $\mathrm{CH}$ stars are confirmed to be binaries, however some $\mathrm{CH}$ stars show carbon isotopic ratios larger than 100 or more. The former stars can be explained if, after accretion from the companion, the star experiences some mixing mechanism (in addition to the first dredge-up) which exposes the accreted material to the $\mathrm{CN}$ cycle and reduces the ${ }^{12} \mathrm{C} /{ }^{13} \mathrm{C}$ ratio keeping $\mathrm{C} / \mathrm{O}>1$. This mixing mechanism might be the cool bottom process proposed by Wasserburg, Boothroyd, \& Sackmann 


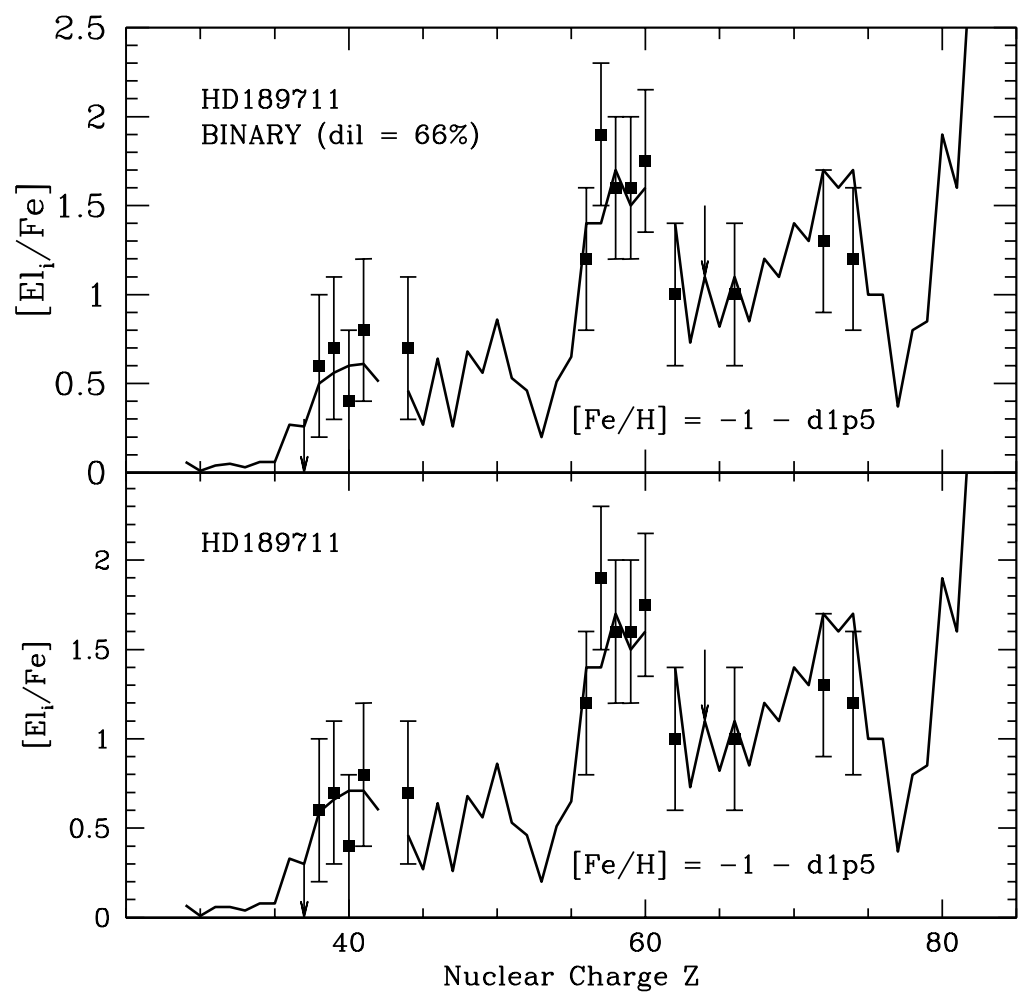

Figure 4 Detailed reproduction of the observed abundances in the $\mathrm{CH}$ star $\mathrm{HD} 189711,[\mathrm{Fe} / \mathrm{H}] \sim-1.0$. The labels indicate the stellar mass model $\left(\mathrm{d} 1 \mathrm{p} 5=1.5 M_{\odot}\right)$ and metallicity that better fits the abundances. The fits are for $\mathrm{C} / \mathrm{O} \sim 1.1$ in the envelope assuming an initial oxygen enhancement $([\mathrm{O} / \mathrm{Fe}] \sim+0.5)$. Upper panel: Extrinsic carbon star fit considering a dilution of the transferred matter by a factor $66 \%$. Lower panel: Intrinsic carbon star fit. Arrows are upper limits. Error bars are indicative.

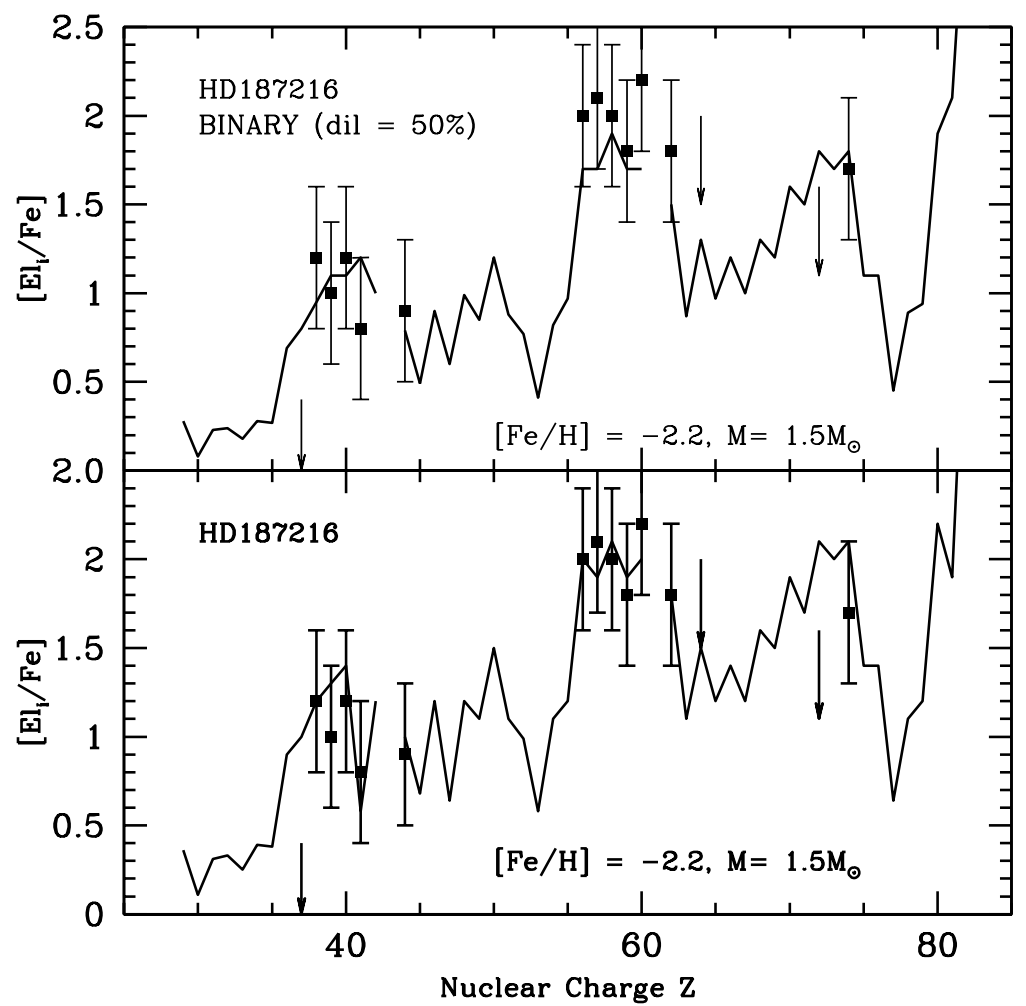

Figure 5 Same as Fig. 4 for the metal-poor $\mathrm{CH}$ star $\mathrm{HD} 187216,[\mathrm{Fe} / \mathrm{H}] \sim-2.0$ at $\mathrm{C} / \mathrm{O} \sim 2.1$. 
Table 2. The Giant Carbon Star Zoo

\begin{tabular}{llclllll}
\hline $\begin{array}{l}\text { Spectral } \\
\text { Type }\end{array}$ & Status & $\begin{array}{c}\text { Extrinsic } \\
\text { Intrinsic }\end{array}$ & ${ }^{12} \mathrm{C} /{ }^{13} \mathrm{C}$ & $\mathrm{Li}$ & s-elements & Mass & Mixing \\
\hline R hot & HB & I & $<15$ & No & No & $\sim 1$ & Yes \\
R cool & $?$ & $?$ & $?$ & $?$ & $?$ & $?$ & $?$ \\
J & $?$ & I & $<15$ & Yes & No & $?$ & Yes \\
SC & TP-AGB & I & $<30$ & $?$ & Yes & $\geq 4 ?$ & No \\
N & TP-AGB & I & $10-100$ & $2 \%$ & Yes & $\leq 3$ & Yes \\
CH & HB & E & $<15$ & No & Yes & $\leq 3$ & Yes \\
& TP-AGB & I & $>100$ & No & Yes & $<2$ & No \\
\hline
\end{tabular}

(1995), which increases dramatically as one reduces the metallicity of the star (Boothroyd \& Sackmann 1999). The $\mathrm{CH}$ stars with very large ${ }^{12} \mathrm{C} /{ }^{13} \mathrm{C}$ ratios may be explained instead by the simple dredge-up of ${ }^{12} \mathrm{C}$ into the envelope, similar to what happens in Population I N stars. In Population II objects, TDU is more efficient, very high $\mathrm{C} / \mathrm{O}$ and ${ }^{12} \mathrm{C} /{ }^{13} \mathrm{C}$ ratios can be obtained, and this may lead to high carbon isotope ratios even after mass transfer.

\section{Summary}

We have reviewed the current understanding of carbon stars of different classes in the Galaxy on the basis of theoretical modelling and the stars' observed chemical characteristics and luminosities. In Table 2 we give a short answer to the main topics discussed. Entries with question marks refer to the cases where there is not enough information, or such information as exists is very dubious. In the case of $\mathrm{CH}$ stars, we have considered two cases, depending on whether the star is on the horizontal branch (with low ${ }^{12} \mathrm{C} /{ }^{13} \mathrm{C}$ ratios), or in the TP-AGB phase. In general, carbon stars constitute one of the most fascinating classes of objects to study. Certainly future theoretical and observational work in Galactic (and extragalactic) carbon stars will help to complete (and maybe revise) this table.

\section{Acknowledgments}

This work was based in part on observations collected at the $4.2 \mathrm{~m} \mathrm{WHT}$ and the $2.5 \mathrm{~m}$ NOT operated on the island of La Palma by the RGO at the Spanish Observatory of Roque de los Muchachos of the Instituto de Astrofísica de Canarias, and also on observations collected with the $2.2 \mathrm{~m}$ telescope at the German-Spanish Astronomical Centre, Calar Alto. It was partially supported by the Spanish grants AYA2000-1574, AYA2002-04094-C03-03, FQM292, by the Italian MURST-Cofin2000 project 'Stellar Observables of Cosmological Relevance', MURST-FIRB 2003 project 'Astrophysical Origin of the Heavy Elements Beyond $\mathrm{Fe}$ ' and by the French-Spanish International Program for Scientific Collaboration, PICASSO HF2000-0087.

\section{References}

Abia, C. et al. 2003, in preparation

Abia, C., Domínguez, I., Gallino, R., Busso, M., Masera, S., Straniero, O., de Laverny, P., Plez, B., \& Isern, J. 2002, ApJ, 579,817
Abia, C., Busso, M., Gallino, R., Domínguez, I., Straniero, O., \& Isern, J. 2001, ApJ, 559, 1117

Abia, C., \& Isern, J. 2000, ApJ, 536, 438

Abia, C., \& Isern, J. 1997, MNRAS, 289, L11

Abia, C., Boffin, H. M. J., Rebolo, R., \& Isern, J. 1993, A\&A, 272,455

Abia, C., \& Wallerstein, G. 1989, MNRAS, 293, 89

Amari, S., Nittler, L. R., Zinner, E., \& Lewis, R. S. 1997, Lunar Planet. Sci., 28, 33

Aoki, W., \& Tsuji, T. 1997, A\&A, 317, 845

Barbaum, C., Morris, M., \& Kahane, C. 1995, ApJ, 450, 862

Beer, H., \& Macklin, R. L. 1989, ApJ, 339, 962

Bergeat, J., Knapik, A., \& Rutily, B. 2002, A\&A, 390, 967

Boothroyd, A. I., \& Sackmann, I. 1999, ApJ, 510, 232

Busso, M., Nollet, K. M., \& Wasserburg, G. J. 2002, in Proceedings of the 11th Workshop on Nuclear Astrophysics, eds. Hillebrandt, W., \& Müller, E. Max-Planck-Institut für Astrophysik p. 18

Busso, M., Lambert, D. L., Gallino, R., Travaglio, C., \& Smith, V. V. 2001, ApJ, 557, 802

Busso, M., Gallino, R., \& Wasserburg, G. J. 1999, ARA\&A, 37,239

Cameron, A. G. W., \& Fowler, W. A. 1971, ApJ, 161, 111

Charbonnel, C. 1995, ApJ, 453, L41

Charboneel, C., \& Balachandran, S. 2000, A\&A, 359, 563

Claussen, M. J., Kleinmann, S. G., Joyce, R. R., \& Jura, M. 1987, ApJS, 65, 385

Cole, P. W., \& Deupree, R. G. 1980, ApJ, 239, 284

de Laverny, P., \& Gustafsson, B. 1999, A\&A, 346, 520

de Laverny, P., \& Gustafsson, B. 1998, A\&A, 332, 661

Dominy, J. 1984, PASP, 97, 1104

Frost, C. A., Cannon, R. C., Lattanzio, J. C., Wood, P. R., \& Forestini, M. 1998, A\&A, 332, L17

Gallino, R., Arlandini, C., Busso, M., Lugaro, M., Travaglio, C., Straniero, O., Chieffi, A., \& Limongi, M. 1998, ApJ, 497, 388

Gilroy, K., \& Brown, J. A. 1991, ApJ, 371, 578

Gratton, R., Sneden, C., Carretta, E., \& Bragaglia, A. 2000, A\&A, 354,169

Gustafsson, B., \& Ryde, N. 1996, in IAU Symposium No. 177. ed. R. Wing, p.481

Harris, M. J., Lambert, D. L., Hinkle, K. H., Gustafsson, B., \& Eriksson, K. 1987, ApJ, 316, 294

Hatzidimitriou, D., Morgan, D. H., Cannon, R. D., \& Croke, B. F. W. 2003, MNRAS, in press

Hauschildt, P. H., Allard, F., Ferguson, J., Baron, E., \& Alexander, D. R. 1999, ApJ, 525, 871

Iben, I. Jr., \& Renzini, A. 1983, ARA\&A, 21, 271

Johnson, H. R. 1982, ApJ, 260, 254

Jorissen, A., Frayer, D. T., Johnson, H. R., Mayor, M., \& Smith, V. V. 1993, A\&A, 271, 463

Keenan, P. C. 1993, PASP, 105, 905

Keenan, P. C., \& Boeshaar, P. C. 1980, ApJS, 43, 379

Kipper, T., Jörgensen, U. G., Klochkova, V. G., \& Panchuk, V. E. 1996, A\&A, 306, 489

Kipper, T., \& Jörgensen, U. G. 1994, A\&A, 290, 148 
Knapp, G. R., Pourbaix, D., \& Jorissen, A. 2001, A\&A, 371, 222 Lambert, D. L., Gustafsson, B., Eriksson, K., \& Hinkle, K. H. 1986, ApJS, 62, 373

Le Bertre, T. 1997, A\&A, 176, 107

Little, S. J., Little-Marenin, I. R., \& Hagen, W. 1987, AJ, 94, 981

Lorenz-Martins, S. 1996, A\&A, 314, 209

Luck, R. E., \& Bond, H. E. 1991, ApJS, 77, 515

McClure, R. D. 1997, PASP, 109, 256

McClure, R. D., \& Woodsworth, A.W. 1990, ApJ, 352, 709

Mengel, J. G., \& Gross, P.G. 1976, Astroph., \& Space Sci., 41, 70

Nittler, L. R. et al. 1995, ApJ, 453, L25

Nollett, K. M., Busso, M., \& Wasserburg, G. 2003, ApJ, 582, 1036

Ohnaka, K., \& Tsuji, T. 1998, A\&A, 335, 1018

Ohnaka, K., \& Tsuji, T. 1996, A\&A, 310, 933

Parthasarathy, M. 1999, in IAU Symposium No. 191, eds. T. Le Bertre, A. Lébre and C. Waelkens, p. 475

Pavlenko, Y. 2003, Astron. Reports, 59, 67

Plez, B. 1999, in Asymptotic Giant Branch Stars, IAU Symp. 191, eds. T. Le Bertre, A. Lèbre, \& C. Waelkens, (San Francisco: ASP), 75

Pourbaix, D., Knapp, G. R., Platais, I., \& Jorissen, A. 2003, A\&A, (in press)
Sackmann, I. J., \& Boothroyd, A. I. 1992, ApJ, 393, L21

Schöier, F. L., \& Olofsson, H. 2000, A\&A, 359, 586

Schwarzschild, M., \& Härm, R. 1965, ApJ, 145, 496

Smith, V. V., \& Lambert, D. L. 1990, ApJS, 72, 387

Straniero, O., Gallino, R., Busso, M., Chieffi, A., Raiteri, C. M., Salaris, M., \& Limongi, M. 1995, ApJ, 440, L85

Utsumi, K. 1985, in Cool Stars with Excesses of Heavy Elements, eds. M. Jascheck \& P. C. Keenan (Dordrecht: Reidel), p. 243

Utsumi, K. 1970, PASJ, 22, 93

Vandervort, G. L. 1958, AJ, 63, 477

van Loon, J. Th. et al. 1998, A\&A, 329, 169

Van Winckel, H., \& Reyniers, C. 2000, A\&A, 354, 135

Vanture, A. D. 1992a, AJ, 103, 2035

Vanture, A. D. 1992b, AJ, 104, 1997

Vanture, A. D. 1992c, AJ, 104, 1986

Wallerstein, G. 1989, ApJS, 91, 341

Wallerstein, G., \& Knapp, G. R. 1998, ARA\&A, 36, 369

Wasserburg, G. J., Boothroyd, A. I., \& Sackmann, I.-J. 1995, ApJ, 440, L101

Weiss, A., Denissenkov, P. A., \& Charbonnel, C. 2000, A\&A, 356,181

Zinner, E. 1998, ARE\&PS, 26, 147 